\title{
Curing the Self-Force Runaway Problem in Finite-Difference Integration
}

\author{
Assaf Lanir, Amos Ori and Orr Sela \\ Department of physics, Technion-Israel Institute of Technology, Haifa 32000, Israel
}

\begin{abstract}
The electromagnetic self-force equation of motion is known to be afflicted by the so-called runaway problem. A similar problem arises in the semiclassical Einstein's field equation and plagues the self-consistent semiclassical evolution of spacetime. Motivated to overcome the latter challenge, we first address the former (which is conceptually simpler), and present a pragmatic finite-difference method designed to numerically integrate the self-force equation of motion while curing the runaway problem. We restrict our attention here to a charged point-like mass in a one-dimensional motion, under a prescribed time-dependent external force $F_{\text {ext }}(t)$. We demonstrate the implementation of our method using two different examples of external force: a Gaussian and a $\operatorname{Sin}^{\wedge} 4$ function. In each of these examples we compare our numerical results with those obtained by two other methods (a "Dirac-type" solution and a "reduction-of-order" solution). Both external-force examples demonstrate a complete suppression of the undesired runaway mode, along with an accurate account of the radiation-reaction effect at the physically relevant time scale - thereby illustrating the effectiveness of our method in curing the self-force runaway problem.
\end{abstract}

\section{INTRODUCTION}

This paper deals with the effect of radiation reaction on the motion of a charged point-like mass. We develop here a pragmatic method to numerically integrate the self-force equation of motion while overcoming the runaway problem. Although, the main motivation for this investigation emerges from an analogous (but considerably more complicated) problem in semiclassical gravity: The self-consistent evolution of spacetime geometry in response to the renormalized stress-energy tensor of quantum fields living in spacetime. In this semiclassical problem too, one encounters a runaway problem [1] 6]; and the technique developed here (in the charged particle context) will potentially be helpful for addressing the analogous semiclassical problem.

Let us first discuss the runaway problem in the case of a charged object: In a Newtonian framework, when a charged point-like mass is accelerated by some external force $\mathbf{F}_{\text {ext }}(t)$, it experiences a radiation-reaction force (or self force), also known as the Abraham-Lorentz force ${ }^{1}$. It is given by [9]

$$
\mathbf{F}_{\text {rad }}=\frac{2 q^{2}}{3 c^{3}} \frac{d \mathbf{a}}{d t},
$$

where $\mathbf{a}$ is the acceleration 3 -vector, $q$ is the charge and $c$ is the speed of light. ${ }^{2}$ The motion of such a charged object therefore satisfies the equation of motion (EOM)

$$
\mathbf{a}(t)=\frac{\mathbf{F}_{e x t}(t)}{m}+\theta \frac{d \mathbf{a}(t)}{d t},
$$

where $m$ is the mass and $\theta \equiv 2 q^{2} / 3 m c^{3}$. Note that (apart from a factor $2 / 3$ ) $\theta$ is the light-travel time across the object's "classical radius" $r_{c} \sim q^{2} / m c^{2}$. The latter is the radius at which the electrostatic enrergy would become comparable to the object's rest mass $m c^{2}$ (should the object's size be smaller than $r_{c}$ ).

Despite of some potentially-confusing aspects it entails, the radiation-reaction force (1.1) is in itself a well-established physical phenomenon. It acts on charged elementary particles as well as on macroscopic charged objects ${ }^{3}$. Yet the resultant EOM (1.2) suffers from an interesting physical-mathematical pathology, known as the runaway problem, which we now briefly discuss.

In typical physical situations the pre-factor $\theta$ is tiny, therefore from the physical viewpoint one expects the radiationreaction phenomenon to only have a small perturbative effect on the object's motion. However, from the pure

\footnotetext{
1 This Newtonian expression has a straightforward relativistic extension, known as the Abraham-Lorentz-Dirac force 7 . 9] (in which a term quadratic in the acceleration is also added). The extension of the analysis below to the relativistic case is straightforward, but not needed here.

2 Throughout this paper we use Gaussian-cgs units.

3 This applies as long as the object's size is small compared to the typical wavelength of the electromagnetic waves it produces. (For example, it would be a perfectly valid approximation for a slightly-charged satellite in orbit around earth.)
} 
mathematical viewpoint the status of the radiation-reaction term looks very different: Regardless of how small $\theta$ is, the term $\theta d \mathbf{a} / d t$ (for any $\theta \neq 0$ ) always constitutes the principal part of the ordinary differential equation (ODE) (1.2). Even with the absence of any external force, the general solution of Eq. 1.2 describes an exponential growth, $\mathbf{a}(t)=\mathbf{a}_{0} \exp (t / \theta)$, where $\mathbf{a}_{0}$ is an arbitrary vector. The presence of an external force $\mathbf{F}_{\text {ext }}$ (assumed here to depend on $t$ only) does not change this situation much, now the general solution is

$$
\mathbf{a}(t)=\mathbf{a}_{\text {spec }}(t)+\mathbf{a}_{0} \exp (t / \theta)
$$

where $\mathbf{a}_{\text {spec }}(t)$ is some specific solution. For a generic solution (generic $\mathbf{a}_{0}$ ) the acceleration grows exponentially at large $t$, with a time scale $\theta$.

The absurd nature of this exact mathematical solution is made especially clear when considering the limit of vanishing $\theta$. From the physical viewpoint we would certainly expect the radiation-reaction effect to vanish at this limit; but the exact mathematical solution shows a very different picture: The rate of exponential blow-up actually speeds up as $\theta$ decreases, and diverges as $\theta \rightarrow 0$. This exponential acceleration of a charged object, and especially its $\propto \theta^{-1}$ divergence, is the essence of the runaway problem.

For illustration, we mention here the value of $\theta$ for two very different physical objects: (i) The electron has $\theta \simeq$ $6.3 \cdot 10^{-24}$ sec. (ii) The sun is claimed to be charged [10] by $q \approx 77$ Coulombs, and correspondingly $\theta \simeq 6.6 \cdot 10^{-43}$ sec. Certainly such an exponentially-growing acceleration of the sun is strikingly inconsistent with our daily astronomical observations.

The aforementioned runaway pathology brings about two different types of challenges: The first is how to reconcile, at the level of principle, the weird mathematical properties of Eq. 1.2 with our basic physical expectations (and our daily experience!) concerning the motion of charged objects. The second one is more pragmatic: How to produce the physically-valid and meaningful solutions for a $(t)$ out of this mathematically-problematic EOM (1.2). Whereas the main objective of this paper is the second challenge, we first briefly discuss the first one.

The expression (1.1) for the radiation-reaction force may be derived in various methods. For the sake of the present discussion we find it useful to consider the extended-object approach [11, which is carried entirely in the framework of classical electrodynamics. In this approach, one considers a charged object of typical size $L$ and computes the overall (classical) electromagnetic force acting on this object, by appropriately integrating the mutual forces acting on the object's various volume elements. Then one takes the limit where the object's size L shrinks to zero. This yields the relativistic Abraham-Lorentz-Dirac equation - which in the Newtonian framework reduces to Eq. (1.1). This method reveals two subtleties that must be kept in mind: (a) The expression (1.1) (like its relativistic counterpart) is only obtained at the $L \rightarrow 0$ limit. For any finite $L$ there are small finite-size corrections. The typical magnitude of these corrections is $\sim t_{L} / t_{\text {orb }}$ where $t_{L} \equiv L / c$ is the light-travel time across the object. Here $t_{\text {orb }}$ denotes the typical dynamical time scale characterizing the object's motion (namely the typical time scale for changing the velocity, acceleration, etc.). For a wide range of object's parameters $L, m, q$, and external forces $\mathbf{F}_{\text {ext }}$, these finite-size corrections are negligible (for a physically valid orbit), but not strictly vanishing. (b) For orbits in which $t_{\text {orb }}$ is small compared to $t_{L}$, the expression (1.1) becomes invalid (as in fact implied from point a).

The reason for the failure of the naive mathematical solutions $(1.3)$ of the EOM $(1.2)$ now becomes clear: In such exponentially-accelerating solutions $t_{\text {orb }}$ becomes $\theta$, which always fails to be $\gg t_{L} .{ }^{4}$ For example, for the charged sun $t_{L} / \theta$ is $\approx 7 \cdot 10^{42}$ rather than $\ll 1$ (for the electron it is of order unity if we take $L$ to be its classical radius). Hence the Abraham-Lorentz force formula is totally invalid for such solutions - explaining the physical irrelevance of the runaway solutions.

The above discussion clarifies the nature of the solutions of the EOM 1.2 that would have physical relevance: First, only solutions with sufficiently large $t_{\text {orb }}$ (compared to $t_{L}$ ) would be relevant. Second, we should not expect the actual physical orbit to be an exact solution of the mathematical EOM: We do expect small deviations (in the actual radiation-reaction force) of typical order $t_{L} / t_{\text {orb }} \ll 1$. Third, in principle the physical solutions should be causal. Namely, the value of $\mathbf{F}_{\text {ext }}$ at a given moment should not affect the orbit at earlier times.

The case that mostly concerns us in this paper is that of weakly charged objects, yielding small $\theta$ (this is the case in which the runaway problem becomes most acute). As it turns out, in this case there is a class of physically-meaningful solutions that naturally suggests itself, which satisfies all the above physical requirements: These are the solutions in which the self force only yields a tiny correction to the object's orbit. Namely, the deviation of the actual a $(t)$ from $\mathbf{F}_{\text {ext }} / m$ is small. In these solutions the orbit's time scale $t_{\text {orb }}$ is just the one set by the external force, which we denote by $\tau$ [namely, the characteristic time scale for significant changes in $\mathbf{F}_{\text {ext }}(t)$ ]. The assumption of "small $\theta$ " can now

\footnotetext{
4 The situation $\theta \gg t_{L}$ would require the object's size $L$ to be $\ll r_{c}$, which would in turn imply that the object's bare mass is negative - which is presumably impossible for a classical object. (It should be emphasized that our mass parameter $m$ is the object's observed mass, and the bare mass is obtained by subtracting the object's electrostatic energy.)
} 
be formulated more explicitly: We shall hereafter assume that $\theta \ll \tau$. This separation of time scales turns out to be essential in the treatment of the runaway problem.

Let us briefly summarize the discussion so far, concerning the motion under weak radiation reaction $(\theta \ll \tau)$ : The generic, exact, mathematical solution of the EOM (1.2) runs-away exponentially, as seen in Eq. (1.3), with an exponential time scale $t_{\text {orb }}=\theta$. However, since this time scale fails to be $\gg t_{L}$, these runaway solutions are physically invalid (because the Abraham-Lorentz formula then fails to describe the correct radiation-reaction force). But there is another class of (approximate) non-runaway solutions. In these solutions $t_{\text {orb }} \sim \tau$ (which is presumably $\gg t_{L}$ ), hence the Abraham-Lorentz expression (1.1) is valid. These are the only (approximate) solutions of Eq. (1.2) which are of physical relevance. ${ }^{5}$ We emphasize again that from the physical viewpoint there is no reason to insist on exact solutions to the EOM (1.2), due to the inherent finite-size error in the radiation-reaction term. This error is of typical order $t_{L} / t_{\text {orb }}$, which in the physically-relevant solutions becomes $t_{L} / \tau \ll 1$ (and is always $\gtrsim \theta / \tau$ ).

After we clarified the nature of the desired radiation-reaction solutions, we come to the next challenge: How to construct these physically relevant solutions from the mathematically-problematic EOM (1.2)? Addressing this question is the main goal of this paper. Note that this is not a trivial task, because the actual mathematical solutions of this ODE diverge exponentially at large $t$ (except for a subclass of measure zero - the Dirac-type solutions discussed below - which however turns out to be a-causal).

Our working assumption here is that at the end of the day it will be necessary to integrate the EOM numerically (while overcoming the runaway problem). Whereas for simple examples of external forces $\mathbf{F}_{\text {ext }}(t)$ an analytical solution may be possible, once $\mathbf{F}_{\text {ext }}$ is allowed to depend on location the situation changes and an analytical solution becomes unlikely. Furthermore, in the problem which really motivates this investigation - the self-consistent semiclassical evolution of spacetime - the solution of the field equation (the semiclassical Einstein equation) ought to be numerical.

Correspondingly, our approach will be to handle the runaway problem while numerically integrating the EOM $[1.2]$, using the finite-difference approach. Recall that the desired physical solutions for the orbit should have a time scale $t_{\text {orb }} \sim \tau$, which is presumably larger than the runaway time-scale $\theta$ by many orders of magnitude. Furthermore, we have no intention to numerically reproduce the (unphysical) runaway dynamics at the timescale $\theta$ : Instead, we would like our numerical integration procedure to simply kill this undesired runaway. As we shall show below, this goal can be naturally achieved if we take the numerical time-step $\delta t$ to be in the range $\theta \ll \delta t \ll \tau$. ${ }^{6}$ We shall design our finite-difference scheme so as to entirely prevent the growth of the undesired runaway mode, while accurately accounting for the radiation-reaction effect at the physically relevant time scale $\tau$.

As was already mentioned above, the physical problem which really motivates this work is the self-consistent semiclassical evolution of e.g. an evaporating macroscopic black hole. In this case spacetime evolution is governed by the semiclassical Einstein equation

$$
G_{\mu \nu}\left(g_{\alpha \beta}\right)=8 \pi G\left\langle\hat{T}_{\mu \nu}\left(g_{\alpha \beta}\right)\right\rangle
$$

where the unknown $g_{\alpha \beta}$ is the spacetime metric, $G_{\mu \nu}$ is the Einstein tensor, $G$ is Newton's gravitational constant, and $<\hat{T}_{\mu \nu}>$ is the (expectation value of the) renormalized stress-energy tensor. This equation describes the back-reaction of a quantum field on spacetime. Recall that the Einstein tensor $G_{\mu \nu}$ is a second-order differential operator acting on $g_{\alpha \beta}$. The right-hand side, being proportional to $\hbar$, is supposed to be a small correction term; however, it contains higher-order derivatives of the metric up to forth order, ${ }^{7}$ formally leading to a runaway instability in the semiclassical metric evolution. This runaway problem is regarded [14] as one of the most serious obstacles in the attempt to obtain the physically relevant self-consistent solutions of the semiclassical Einstein equation (1.4).

As was pointed out in Ref. [1, there is a remarkable analogy between the two problems: In both cases, we originally have a second-order differential equation [for the particle's orbit $\mathbf{r}(t)$ or for the spacetime metric $g_{\alpha \beta}(x)$ ]; and there is a back-reaction term which is multiplied by a small pre-factor, yet it includes higher-order derivatives. In both problems, therefore, there is a runaway catastrophe: The general mathematical solution of the back-reaction equation blows up exponentially with a ridiculously small time scale (which becomes shorter as the pre-factor of the back-reaction term decreases). In both cases the relevant physical solutions are those with dynamical time scales much longer than the

\footnotetext{
5 There also exist the class of "Dirac-type" (described below), which are exact solutions of the EOM 1.2 with no runaway. But these solutions are a-causal.

${ }^{6}$ The condition $\delta t \ll \tau$ is necessary for achieving an accurate numerical solution (at the relevant time-scale $\tau$ ). Eventually it turns out that the other condition $\delta t \gg \theta$ is not really necessary, it may be replaced by the weaker one $\delta t>2 \theta$ (see Sec. II).

7 The source term $\left\langle\hat{T}_{\mu \nu}\right\rangle$ is basically made of the contributions of the various modes of the quantum field. However, the sum/integral over the modes diverges, and requires renormalization. In curved spacetime the renormalization is usually done by point-splitting, and it involves the subtraction of certain counter-terms [12, 13, made of the Riemann (or Ricci) tensor and its derivatives up to second order. This amounts to fourth-order derivatives of the basic unknown $g_{\alpha \beta}$.
} 
runaway timescale. Owing to these similarities, we anticipate that the finite-difference method developed here will successfully cure the runaway problem in the semiclassical context as well.

In the charged-object case, in order to obtain physically meaningful solutions to Eq. 1.2 , two different approaches have been introduced previously. Both approaches are further described and implemented in Sec. III below, in order to compare them with our new finite-difference method. The first, introduced by Dirac 7, assumes that the external force should vanish at late time. Correspondingly it considers only the solutions to Eq. 1.2 for which a $(t)$ vanishes at late time. This prescription inherently violates causality, but nevertheless the time scale of the violation is minuscule, it coincides with the runaway timescale $\theta$ (e.g. for the electron it is $\sim 6 \cdot 10^{-24} s$ ). The second approach is the method of reduction of order [8, 9, 15, 16. In this method, one replaces $d \mathbf{a} / d t$ in Eq. 1.2 with the time derivative of the external force (see Sec. III). Note that this procedure involves neglecting a term quadratic in the small parameter $\theta / \tau$.

Both approaches, Dirac's method and reduction of order, seem to be inapplicable (or at least impractical) in the problem of self-consistent semiclassical evolution of e.g. an evaporating black hole. Dirac's method relies on requiring a trivial asymptotic behavior of the solution at the infinite future. This seems to be inapplicable to semiclassical evolution of black holes, due to the presence of a future singularity inside the horizon. In addition, since we do not have at our disposal a closed-form solution of the semiclassical Einstein equation, there is no way to know in advance which initial conditions would lead to a desired late-time solution (even if such a desired solution existed). The method of reduction of order works well in the case of ODEs like Eq. (1.2), in which there is a single higher-order derivative term $(d \mathbf{a} / d t)$ that needs be eliminated, which can be directly obtained by differentiating the "original" EOM $a=\mathbf{F}_{\text {ext }} / \mathrm{m}$. This is not the case in our semiclassical problem, mainly because the semiclassical Einstein equation (1.4) is a partial differential equation (PDE), making it hard to eliminate the various higher-order derivative terms involved in $\left\langle\hat{T}_{\mu \nu}\right\rangle$. We point out that the method of reduction of order has been applied to several semiclassical gravitational systems (see, for example, 17, 18); but to the best of our knowledge, in all these cases either (i) the semiclassical Einstein equation reduced to an ODE, or (ii) the quantum field was a conformal scalar field. (In the latter case no derivatives of the Riemann tensor appear in the counter-term. Only derivatives of the Ricci scalar and Ricci tensor appear, which can in turn be expressed by the energy-momentum tensor via Einstein equation.) Both simplifying factors (i) or (ii) allow for easy reduction of order. This leaves unresolved the problem of doing reduction of order in the situation of e.g. black-hole evaporation (via quantum fields other than conformal scalar field).

On the other hand, the finite-difference method introduced here will hopefully be applicable to the problem of semiclassical black hole evaporation as well. Of course this problem is technically much harder than the electromagnetic radiation reaction problem, due to several reasons. One obvious difference is that the semiclassical problem involves PDEs rather than ODEs. As far as we can foresee, this difference should not disable our finite-difference method (although it may possibly make its application technically harder). ${ }^{8}$

Throughout this paper we shall restrict our attention to one-dimensional motion, and also to a prescribed external force $\mathbf{F}_{\text {ext }}(t)$ that depends on time only. In a forthcoming paper [19] we extend the method to an external force depending on space as well as time, yielding a third-order radiation-reaction EOM in terms of the position (rather than a first-order equation for the acceleration). We shall show that our finite-difference method works very well in this generalized framework as well. This will be carried out as an intermediate stage towards the application of the procedure to a hyperbolic PDE with an artificially-added higher-derivative term - before applying it finally to the semiclassical Einstein field equation.

The rest of this paper is organized as follows. In Sec. II we construct the finite-difference scheme which resolves the runaway problem. In Sec. III we numerically implement our finite-difference method to two examples of external force, one is Gaussian and the other is of a $\operatorname{Sin}^{\wedge} 4$ form. We compare our numerical results with the corresponding Dirac solution and the solution obtained by reduction of order, and we find good agreement. We then summarize our results and give some concluding remarks in Sec. IV]

\section{DISCRETIZING THE EQUATION OF MOTION}

Our starting point is the Newtonian EOM (1.2), which we take now to be one-dimensional (say in the $x$ direction):

$$
a(t)=\frac{F_{e x t}(t)}{m}+\theta \frac{d a(t)}{d t},
$$

\footnotetext{
8 A much more crucial difference is that in the semiclassical problem it is very difficult to compute the source term $<\hat{T}_{\mu \nu}>$. In particular it requires the computation of many field's modes on the background metric $g_{\mu \nu}$, combined with the point-splitting [12] 13] regularization procedure.
} 


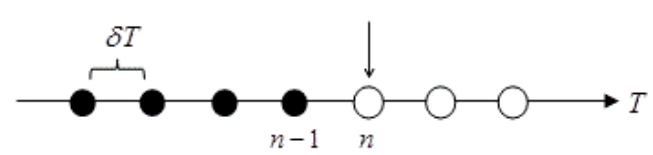

Figure 1: Illustration of the lattice points in the numerical scheme. The full black spots mark the points $k<n$ for which $A_{k}$ has already been computed. The empty circles denote points $k \geq n$ for which the values of $A_{k}$ are still unknown. The vertical arrow points at the $n$th lattice point, where the value of $A$ is being computed at this stage.

The distance between each pair of neighboring points is the "lattice constant" $\delta T$.

where, recall, $\theta \equiv 2 q^{2} / 3 m c^{3}$. We assume that the external force $F_{\text {ext }}$ only depends on $t$, not on $x$. Recall that there are two different time scales in this EOM: the external-force time scale $\tau$, and the runaway time scale $\theta$; and throughout this paper we are interested in the case of weak radiation reaction, $\theta \ll \tau$ (the situation that mostly sharpens the runaway problem.) Our goal is to design a stable numerical discretization scheme for such an EOM, free of runaway.

As a first step, we use the time parameter $\tau$ to transform the variables in Eq. 2.1 into dimensionless ones:

$$
t \rightarrow T \equiv t / \tau, \quad a \rightarrow A \equiv(\tau / c) a, \quad F_{e x t} \rightarrow F \equiv(\tau / m c) F_{e x t}
$$

The resulting dimensionless EOM is

$$
A=\varepsilon \dot{A}+F(T),
$$

where an over-dot denotes $d / d T$, and $\varepsilon \equiv \theta / \tau$. Our assumption of weak radiation reaction now reads $\varepsilon \ll 1$.

In a finite-difference numerical scheme, we treat the time axis as a discrete set of "lattice" points $T_{k}$, with a fixed separation $\delta T=T_{k+1}-T_{k}$ (see Fig. 1). In each step of the numerical computation, the value of $A(T)$ is already known on all lattice points $k<n$ (for some $n$ ), but $A_{n}$ (like all points $A_{k>n}$ ) is still unknown. The numerical scheme should then determine $A_{n}$, expressing it in terms of (some of) the already-known points $A_{k<n}$ (the black spots in Fig. 1). We shall restrict the discussion here to the simplest example for the discretization of a first-order ODE, which we call two-point discretization, that only involves the two lattice points $A_{n-1}$ and $A_{n}$.

We need to (approximately) express the various elements of Eq. 2.2 in terms of the two available data points $A_{n-1}$ and $A_{n}$. There is an obvious expression for the discretized derivative $\dot{A}$ :

$$
\dot{A}(T) \rightarrow \frac{A_{n}-A_{n-1}}{\delta T} .
$$

However, a priori there is no unique choice for the discretization of $A(T)$. For example, one may choose to represent it by $A_{n}$, or $A_{n-1}$, or by some weighted average of the two. Each choice will lead to a slightly different finite-difference scheme. At this stage we find it useful to allow for a general representation

$$
A(T) \rightarrow \gamma A_{n}+(1-\gamma) A_{n-1},
$$

with a (real) free parameter $\gamma$. The representation of $F(T)$ is done similarly, using the same weighted average:

$$
F(T) \rightarrow \gamma F_{n}+(1-\gamma) F_{n-1},
$$

where $F_{n} \equiv F\left(T_{0}+n \delta T\right)$, and $T_{0}$ is the $T$ value corresponding to the first lattice point $n=0$. The discretized (dimensionless) EOM then becomes

$$
\gamma A_{n}+(1-\gamma) A_{n-1}=\epsilon\left(A_{n}-A_{n-1}\right)+\left[\gamma F_{n}+(1-\gamma) F_{n-1}\right],
$$

with a free discretization parameter $\gamma$, where $\epsilon \equiv \varepsilon / \delta T$.

\section{A. Choosing the discretization parameter $\gamma$}

As it turns out, the choice of $\gamma$ will affect the dynamical behavior of the finite-difference scheme. We shall take advantage of this free parameter, in order to achieve our goal of suppressing the problematic runaway mode.

Let $A_{\text {phys }}(T)$ denote the solution of the ODE (2.2) which we regard as the desired physical solution (i.e. the one which does not exhibit exponential runaway). Consider now another solution $A(t)$ which (at some moment $T$ ) 
slightly deviates from $A_{\text {phys }}(T)$. We denote the difference by $\Delta A(T) \equiv A(T)-A_{\text {phys }}(T)$. This deviation satisfies the homogeneous equation

$$
\Delta A=\varepsilon \Delta \dot{A} .
$$

Mathematically, every small initial deviation $\Delta A$ from $A_{\text {phys }}(T)$ will grow exponentially in $T$ : Obviously the general solution of Eq. (2.7) is $\Delta A(T)=$ const $\cdot \exp (T / \varepsilon)$. In order for the numerical scheme to stably recover the desired solution $A_{\text {phys }}(T)$, our finite-difference scheme must suppress this homogeneous mode. Applying the above discretization scheme to the homogeneous equation (2.7) yields

$$
\gamma \Delta A_{n}+(1-\gamma) \Delta A_{n-1}=\epsilon\left(\Delta A_{n}-\Delta A_{n-1}\right)
$$

Extracting $\Delta A_{n}$ we obtain

$$
\Delta A_{n}=p \Delta A_{n-1}
$$

where

$$
p=1-\frac{1}{\gamma-\epsilon} .
$$

This formula describes a geometric sequence, $\Delta A_{n}=$ const $\cdot p^{n}$. The qualitative nature of this sequence will depend crucially on whether $|p|$ is greater or smaller than one: The sequence $\Delta A_{n}$ will blow up (as $n \rightarrow \infty$ ) in the first case and decay in the second. We want our finite-difference scheme to converge to the desired solution $A_{\text {phys }}(T)$, which would correspond to $\Delta A(t) \rightarrow 0$ (and the faster the better). The range of $\gamma$ which satisfies this stability condition is $\gamma>\epsilon+1 / 2$.

We find it especially convenient to work with $\gamma=1$ (implying that $A(t)$ is simply discretized as $A_{n}$ ). This choice of $\gamma$ corresponds to the smallest value of $|p|$ (and hence to the quickest damping of $\Delta A_{n}$ ), if: (i) we consider a $\gamma$ choice independent of $\epsilon$, and (ii) we recall that in typical situations we shall have $\epsilon \ll 1$, which (for $\gamma=1$ ) would yield $p \approx-\epsilon$.

\section{B. The final discretization scheme}

Substituting $\gamma=1$ in Eq. 2.6 and extracting $A_{n}$, we obtain the discretized EOM:

$$
A_{n}=-\frac{\epsilon}{1-\epsilon} A_{n-1}+\frac{1}{1-\epsilon} F_{n},
$$

where, recall, $\epsilon \equiv \varepsilon / \delta T$. The deviations from the desired solution $A_{\text {phys }}(T)$ will thus decay according to the geometric sequence

$$
\Delta A_{n}=\text { const } \cdot p^{n}, \quad p=-\frac{\epsilon}{1-\epsilon} .
$$

As was already mentioned in the Introduction, in a typical situation (with a huge separation of time scales $\theta \ll \tau$ ) we would typically choose the numerical time step $\delta t$ in the range $\theta \ll \delta t \ll \tau$. In terms of the dimensionless parameters this reads $\varepsilon \ll \delta T \ll 1$. The condition $\delta T \ll 1$ is really necessary for controlling the truncation error. The other condition $\delta T \gg \varepsilon$ is not mandatory but merely a matter of typicality and convenience. Note, however, that we must always require

$$
\delta T>2 \varepsilon
$$

in order to achieve numerical stability. This directly follows from Eq. (2.11), which indicates that the condition for having $|p|<1$ is $\epsilon<1 / 2$.

The choice of $\delta T$ in the appropriate range $(2 \varepsilon<\delta T \ll 1)$ guarantees that no runaway mode (and no numericalinstability mode) will develop. The evolving numerical solution will therefore be characterized by the external-force time scale; and since the time step is much smaller than that scale, the truncation error will be well controlled and the numerical solution should faithfully recover the desired physical solution. The effectiveness of this numerical procedure will be demonstrated in the next section. 
Initial value for $A$

In principle, in the numerical scheme described above we need to choose the initial value of $A$, which we denote $A_{0}$. Owing to the quick suppression of the homogeneous mode, the resultant numerical solution is insensitive to the choice of $A_{0}$ (except in a very short interval at the beginning, a few times $\delta T$ ). We shall hereafter adopt a standard choice:

$$
A_{0}=F(T=0) .
$$

\section{NUMERICAL IMPLEMENTATIONS}

We shall now demonstrate this finite-difference method numerically, using two different examples of external force $F(T)$. In both examples we shall compare our numerical result with the corresponding Dirac-type solution and with the solution of the reduction-of-order variant of Eq. 2.2. As a preparatory step we shall now develop the analytical expressions for the Dirac-type and the reduction-of-order solutions, for a general $F(T)$.

We begin with the general solution of Eq. 2.2, written in the form

$$
A(T)=-\frac{1}{\varepsilon} e^{T / \varepsilon} \int_{\infty}^{T} e^{-s / \varepsilon} F(s) d s+C e^{T / \varepsilon},
$$

where $C$ is an arbitrary constant. Assuming a function $F(T)$ of a bounded support (or an "effectively-bounded" support as in the Gaussian case), at large $T$ this solution approaches $C e^{T / \varepsilon}$. The Dirac-type solution, which we denote $A_{D}(T)$, is the unique solution that vanishes as $T \rightarrow \infty$ - namely the $C=0$ solution:

$$
A_{D}(T)=-\frac{1}{\varepsilon} e^{T / \varepsilon} \int_{\infty}^{T} e^{-s / \varepsilon} F(s) d s .
$$

Next, we apply the reduction-of-order procedure (to first order in $\varepsilon$ ), and construct the corresponding analytical expression, which we denote $A_{r}(T)$. To this end, we first differentiate Eq. 2.2 with respect to $T$ :

$$
\dot{A}=\varepsilon \ddot{A}+\dot{F}(T) .
$$

Inserting this into the right-hand side of Eq. 2.2 we get

$$
A(T)=\varepsilon[\varepsilon \ddot{A}(T)+\dot{F}(T)]+F(T)=\varepsilon \dot{F}(T)+F(T)+O\left(\varepsilon^{2}\right) .
$$

In constructing the reduction-of-order solution, we simply ignore the $O\left(\varepsilon^{2}\right)$ term and obtain:

$$
A_{r}(T)=F(T)+\varepsilon \dot{F}(T) .
$$

We point out that the Dirac-type solution is in principle a-causal, as clearly indicated by the lower integration limit $s \rightarrow \infty$ in Eq. (3.1) (nevertheless this a-causality is usually small, the advance time is typically of magnitude $\varepsilon$ ). The reduction-of-order expression $A_{r}(T)$ does not suffer from such violation of causality. This expression is not an exact solution of the EOM (2.2), but nevertheless it is a solution to first order in $\varepsilon$.

\section{A. Gaussian External Force}

As a first example, we choose $F(T)$ to be a Gaussian,

$$
F(T)=B \exp \left[-\frac{(T-\mu)^{2}}{2 \sigma^{2}}\right]
$$

as displayed in Fig. 22a. The corresponding Dirac-type solution (3.1) is then

$$
A_{D}(T)=\frac{B}{\varepsilon} \sqrt{\frac{\pi \sigma^{2}}{2}} \exp \left(\frac{T}{\varepsilon}+\frac{\sigma^{2} / \varepsilon-2 \mu}{2 \varepsilon}\right) \operatorname{Erfc}\left(\frac{\sigma^{2} / \varepsilon+T-\mu}{\sqrt{2 \sigma^{2}}}\right),
$$


where Erfc is the complementary error function given by

$$
\operatorname{Erfc}(x)=\frac{2}{\sqrt{\pi}} \int_{x}^{\infty} \exp \left(-s^{2}\right) d s .
$$

The reduction-of-order solution 3.2 now reads

$$
A_{r}(T)=\left[1-\frac{\varepsilon}{\sigma^{2}}(T-\mu)\right] B \exp \left[-\frac{(T-\mu)^{2}}{2 \sigma^{2}}\right] .
$$

Specifically we choose here the parameters $B=\sigma=1$ and $\mu=10$. For the radiation-reaction strength we choose $\varepsilon=10^{-3}$. We then solve Eq. (2.2) numerically using the finite-difference scheme constructed above, Eq. (2.10).

Figure 2 displays this numerical solution, clearly indicating that no runaway problem occurs. Note that panel (a) of this figure simultaneously shows the force $F(T)$ and the resultant acceleration $A(T)$ (the latter actually comes in three variants as we shortly detail); but these two quantities are indistinguishable in this graph because the radiationreaction magnitude is very small, $\varepsilon=10^{-3}$. To clearly see the radiation-reaction effect we need to look at the difference between $A$ and $F$. To this end, panel (b) displays the quantity $\alpha(T) \equiv A(T)-F(T)$ (which is entirely due to radiation reaction).

More specifically, the figure presents three different variants of $A$ (and $\alpha$ ): our numerically-constructed solution $A_{N}(T)$, and the two analytically-constructed solutions used here for comparison, namely the Dirac-type solution $A_{D}(T)$ and the reduction-of-order solution $A_{r}(T)$ [Eqs. (3.4) and (3.5) respectively]. These three variants of $A$ yield three corresponding variants of $\alpha=A-F$, denoted $\alpha_{N}, \alpha_{D}$, and $\alpha_{r}$ respectively. Panel (b) shows that our numericallyconstructed solution $\alpha_{N}$ is visually-indistinguishable from the two other, analytically constructed, variants $\alpha_{D}$ and $\alpha_{r}$ (the difference between the three variants is presented below in Fig. 3). This indicates that our finite-difference scheme accurately captures the effect of the radiation-reaction force on the acceleration, despite the small value of $\varepsilon$ used here.

Figure 3 quantifies the differences between the three variants of $\alpha$. It displays $\alpha_{r}-\alpha_{D}$ (marked by the dashed purple curve). It also displays $\alpha_{N}-\alpha_{D}$ for three different values of $\delta T$ (used in the construction of $\alpha_{N}$ ): $\delta T_{1}=10^{-2}$, $\delta T_{2}=5 \cdot 10^{-3}$, and $\delta T_{3}=2.5 \cdot 10^{-3}$. It indicates first-order convergence of the finite-difference scheme, as expected. Notice that the smallest numerical time step used here $\left(\delta T_{3}\right)$ is only slightly larger than the minimal allowed value $\left(2 \varepsilon=2 \cdot 10^{-3}\right)$, but the numerical scheme is still stable as $|p|=2 / 3<1$. For this value of $\delta T$, the "numerical error" indicator (namely the deviation of $\alpha_{N}$ from $\alpha_{D}{ }^{9}$ ) is comparable to the difference $\alpha_{r}-\alpha_{D}$.

The analysis in Sec. II indicated that for stable numerical integration we must choose $\delta T>2 \varepsilon$ (to ensure $|p|<1$ ). We checked this criterion numerically: For $\delta T=2.01 \varepsilon$ the numerics worked very well, but for $\delta T=1.99 \varepsilon$ we encountered a severe instability, expressed in an exponentially-growing error. This was found to be the case in both the Gaussian and the $\operatorname{Sin}^{\wedge} 4$ cases.

\section{B. $\operatorname{Sin}^{\wedge} 4$ External Force}

Next we choose the external force $F(T)$ to be the following function:

$$
F(T)=B \sin ^{4}\left[\frac{\pi}{W}(T-\mu)\right] \Theta(T-\mu+W) \Theta(\mu+W-T) .
$$

It represents a full period ( $T$ interval equals $2 W$ ) of a $\operatorname{Sin} \wedge 4$ function centered at $T=\mu$, with amplitude $B$. This function, displayed in Fig. 4 4 , was chosen as an example of a three-times differentiable function with a compact support. The corresponding Dirac-type solution (3.1) is then ${ }^{10}$

$$
A_{D}(T)=-\frac{1}{\varepsilon} e^{T / \varepsilon} \int_{\infty}^{T} e^{-s / \varepsilon} B \sin ^{4}\left[\frac{\pi}{W}(s-\mu)\right] \Theta(s-\mu+W) \Theta(\mu+W-s) d s
$$

\footnotetext{
${ }^{9}$ It is sensible to use $\alpha_{D}$ as a reference for assessing the accuracy of $\alpha_{N}$, because $A_{D}(T)$ is an exact closed-form solution of the EOM.

10 The integral appearing in Eq. 3.7 is elementary and straightforward, yet the explicit expression is rather lengthy (and non-illuminating), therefore we do not present it here.
} 


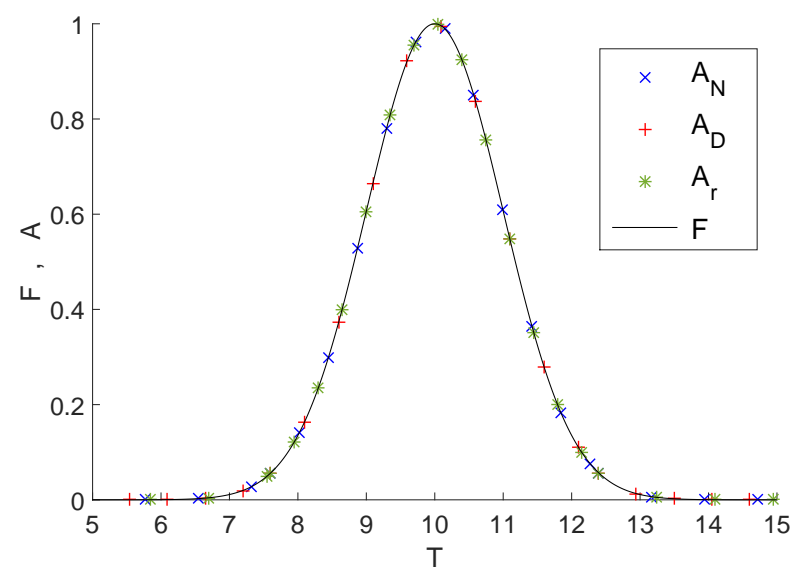

(a)

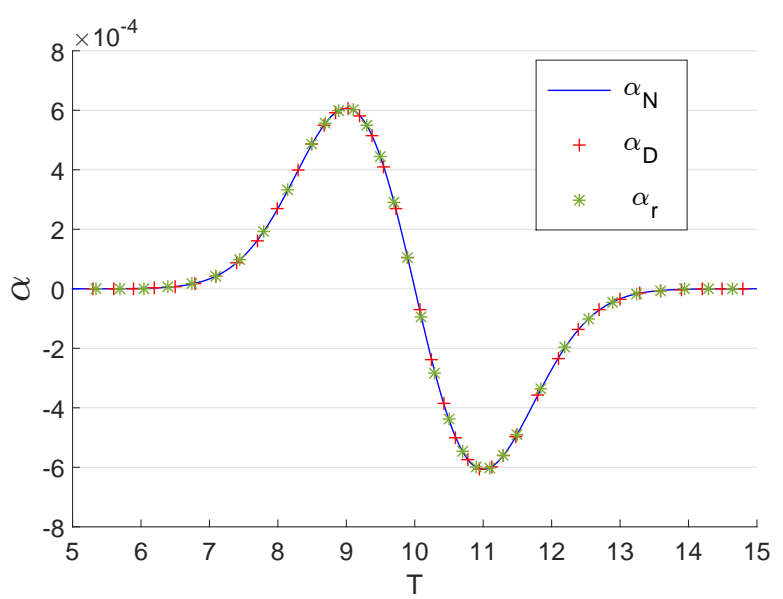

(b)

Figure 2: Gaussian external force. Panel (a) displays the external force $F(T)$ (black solid line) given by Eq. (3.3), with parameters $B=\sigma=1$ and $\mu=10$. It also displays the solution $A(T)$ of the EOM 2.2 corresponding to this Gaussian external force. This solution comes in three variants: Our numerically-constructed solution $A_{N}(T)$, and the two analytically-constructed solutions $A_{D}(T)$ (Dirac-type solution) and $A_{r}(T)$ (reduction of order), used here for comparison. These three variants of $A(T)$ are respectively represented by blue crosses, red "plus" symbols, and green stars. However these three accelerations are visually indistinguishable from the black curve of $F(T)$ (and from each other). Panel (b) displays the differences $\alpha(T) \equiv A(T)-F(T)$ between the external force and the acceleration(s) (which directly represent the effect of the radiation-reaction force). There are three variants of $\alpha$, in correspondence with the three variants of $A$ : The blue solid curve denotes $\alpha_{N}=A_{N}-F$, the red "plus" symbols denote $\alpha_{D}=A_{D}-F$, and the green stars denote $\alpha_{r}=A_{r}-F$. The numerically-constructed function $\alpha_{N}$ is visually

indistinguishable from the two analytically-constructed functions $\alpha_{D}, \alpha_{r}$ - demonstrating the accuracy of our finite-difference scheme. In both panels the numerical time step was $\delta T=5 \cdot 10^{-3}$ (correspondingly the number of steps was $\left.N=4 \cdot 10^{3}\right)$.

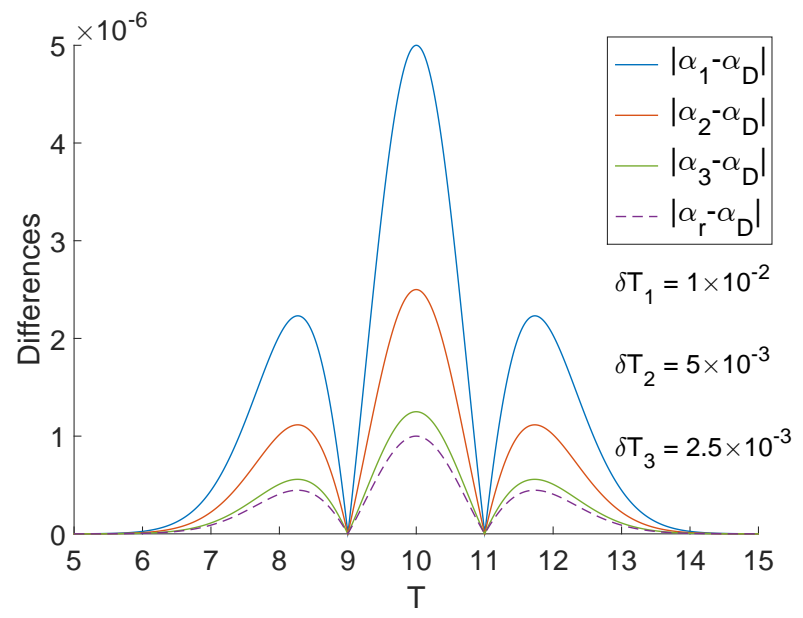

Figure 3: The differences between the three different variants of $\alpha(T)$, for the same Gaussian force as in Fig. 2 .

The dashed purple curve denotes $\left|\alpha_{r}-\alpha_{D}\right|$. The three solid curves all denote $\left|\alpha_{N}-\alpha_{D}\right|$ but for numerically constructed functions $\alpha_{N}(T)$ obtained with three different time steps: $\delta T_{1}=10^{-2}$ (blue), $\delta T_{2}=5 \cdot 10^{-3}$ (orange), and $\delta T_{3}=2.5 \cdot 10^{-3}$ (green). In the legend we use the abbreviated notation $\alpha_{i} \equiv \alpha_{N}\left(\delta T=\delta T_{i}\right)$. 


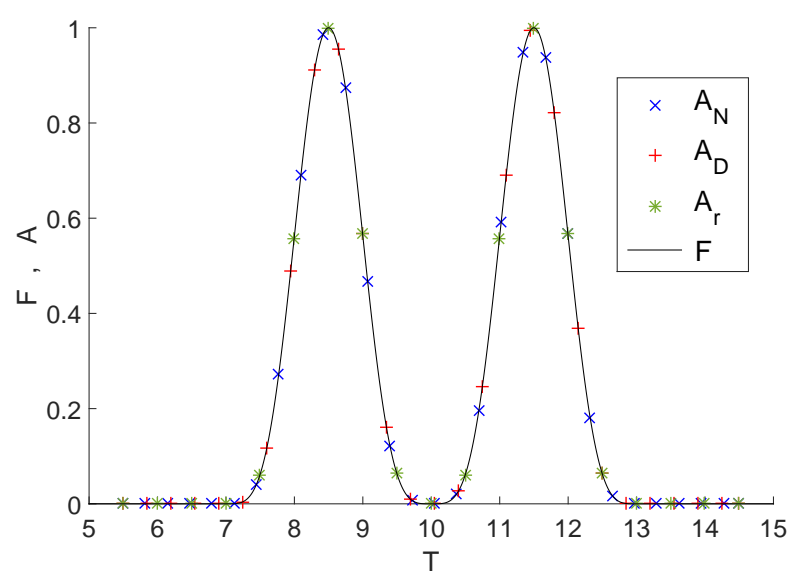

(a)

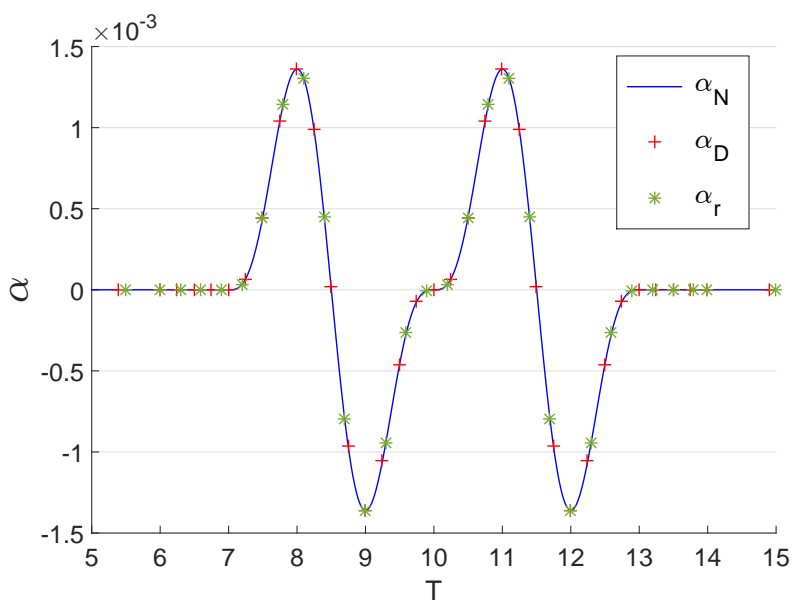

(b)

Figure 4: $\operatorname{Sin}^{\wedge} 4$ external force. The parameters chosen are $B=1, W=3, \mu=10$, and again we use $\varepsilon=10^{-3}$. Presentation and notation is the same as in Fig. 2 above. In particular, panel (a) displays both $F(T)$ and $A(T)$ (indistinguishable), and panel (b) displays $\alpha(T)=A(T)-F(T)$; and as before both $A$ and $\alpha$ come with their three variants, denoted by the indices " $N$ ", " $D$ ", or " $r$ ". All symbols are the same as in the corresponding panels in Fig.

The reduction-of-order solution 3.2 now becomes

$$
A_{r}(T)=B \Theta(T-\mu+W) \Theta(\mu+W-T) \sin ^{4}\left[\frac{\pi}{W}(T-\mu)\right]\left\{1+\varepsilon \frac{4 \pi}{W} \cot \left[\frac{\pi}{W}(T-\mu)\right]\right\}
$$

We choose here the parameters $B=1, W=3$ and $\mu=10$. The radiation-reaction strength parameter is the same as before, $\varepsilon=10^{-3}$. We again solve Eq. 2.2 numerically using the finite-difference scheme constructed in Sec. [II]

The results closely resemble the case of Gaussian force presented above. Figure 4 a (fully analogous to Fig. 2a) displays both $F(T)$ and $A(T)$. The latter again comes in three variants: our numerical solution $A_{N}(T)$, the Dirac-type solution $A_{D}(T)$, and the reduction-of-order solution $A_{r}(T)$. Figure $4 \mathrm{~b}$ (fully analogous to Fig. $2 \mathrm{~b}$ ) depicts the backreaction effect as represented by the difference $\alpha(T)=A(T)-F(T)-$ with its three variants $\alpha_{N}, \alpha_{D}$, and $\alpha_{r}$. Figure 5 (analogous to Fig. 3) shows the differences between these three variants of $\alpha$. In particular, it displays $\alpha_{N}-\alpha_{D}$ for three different values of $\delta T$ (the same values $\delta T_{1,2,3}$ used above). Overall, these graphs lead to the same conclusions as in the case of a Gaussian force: The runaway problem has been entirely cured in the numerical integration, the truncation errors associated with the three time steps $\delta T_{1,2,3}$ are compatible with first-order convergence, and overall we achieve a good accuracy. Again, for the smallest time step $\delta T_{3}$ the difference $\alpha_{N}-\alpha_{D}$ is comparable to $\alpha_{r}-\alpha_{D}$. 


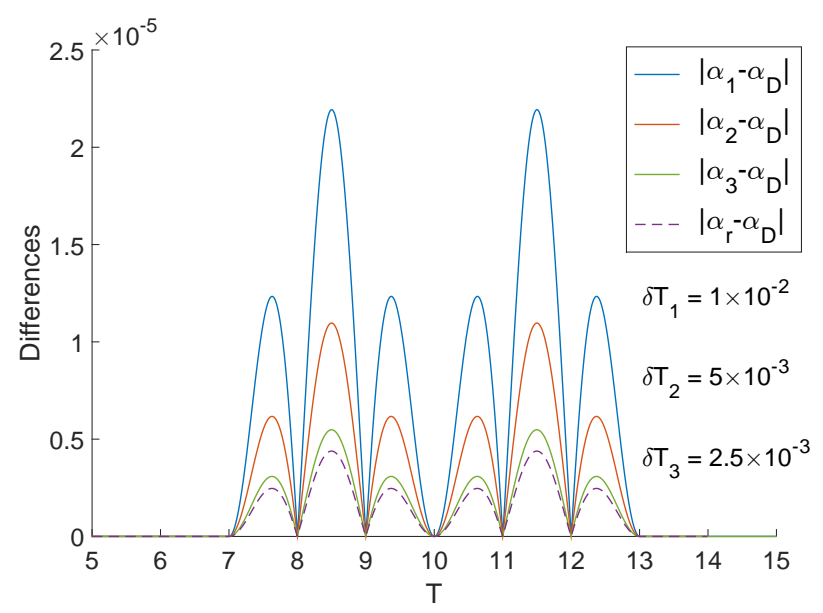

Figure 5: The differences between the three different variants of $\alpha(T)$, for the $\operatorname{Sin}^{\wedge} 4$ external force. Presentation and notation is the same as in Fig. 3 above. The dashed purple curve denotes $\left|\alpha_{r}-\alpha_{D}\right|$. The three solid curves all denote $\left|\alpha_{N}-\alpha_{D}\right|$, for numerically constructed functions $\alpha_{N}(T)$ obtained with the same set of three time steps used above: $\delta T_{1}=10^{-2}$ (blue), $\delta T_{2}=5 \cdot 10^{-3}$ (orange), and $\delta T_{3}=2.5 \cdot 10^{-3}$ (green). In the legend we again use the abbreviated notation $\alpha_{i} \equiv \alpha_{N}\left(\delta T=\delta T_{i}\right)$.

\section{CONCLUSIONS}

In this paper we have presented a pragmatic method to numerically construct the physically meaningful solutions of the self-force equation of motion, thereby overcoming the runaway problem plaguing the analysis of the motion of a charged point-like mass under the influence of radiation reaction. This method exploits the fundamental separation of time scales which usually arises in this problem, by choosing the numerical time-step to be large compared to the runaway time-scale, yet much smaller than the characteristic time scale $\tau$ of the external force (which is also the time-scale of the actual physical orbit). The finite-difference scheme was designed to accurately incorporate the effect of the radiation-reaction force at the physically relevant time scale $\tau$, while completely suppressing the undesired runaway mode. As a demonstration of this finite-difference method, we have numerically computed the time-dependent acceleration of a charged point-like particle subject to two different types of external force $F_{\text {ext }}(t)$, one is Gaussian and the other has the form of $\operatorname{Sin}^{\wedge} 4$. The resulting accelerations accurately match the physically expected ones.

As discussed above, there is a close analogy between the runaway problem associated with electromagnetic radiation reaction, analyzed in this paper, and the one that occurs in semiclassical general relativity, for example in the context of black-hole evaporation. However, the latter turns out to be significantly more involved, as the semiclassical Einstein field equation is a PDE rather than ODE ${ }^{11}$. Still, we expect our finite-difference method to be applicable to this case as well, despite its technically more challenging nature.

The numerical procedure presented here is basically a first-order finite-difference scheme, implying that the numerical error scales like the time step $\delta T$. It may be useful to upgrade the scheme to second-order accuracy. In this regard we point out that there is no much point in increasing the numerical accuracy: In the present scheme the truncation error (e.g. the relative error in $\alpha=A-F$ ) is already at order $\sim \varepsilon=\theta / \tau$; and as explained in the Introduction this is the best one can hope to achieve due to the inherent limited accuracy in the self-force formula (1.1), which originates from finite-size effects. Nevertheless, a second-order numerical scheme will allow increasing $\delta \bar{T}$, which will in turn significantly shorten computation times.

In the published version of this paper [19, which includes a significant extension of this manuscript by A. Lanir and O. Sela, we extend this investigation to an external force depending on space as well as time. In that case, one obtains a third-order radiation-reaction EOM in terms of the position (rather than a first-order equation for the acceleration). We also extend the analysis further to include higher order EOMs. We show that our finite-difference method (when properly generalized) is applicable in this more general framework as well.

\footnotetext{
11 See also footnote 8 above.
} 
Subsequently, the method should be extended to the application on a PDE system with artificial radiation-reactionlike term, in a preparatory step before applying it to the semiclassical Einstein field equation.

[1] E. Flanagan and R. Wald, Does back reaction enforce the averaged null energy condition in semiclassical gravity? , Phys. Rev. D 54, 6233 (1996).

[2] J. Z. Simon, Higher-derivative Lagrangians, nonlocality, problems, and solutions, Phys. Rev. D 41, 3720 (1990).

[3] J. Z. Simon, Stability of flat space, semiclassical gravity, and higher derivatives, Phys. Rev. D 43, 3308 (1991).

[4] J. Z. Simon, No Starobinsky inflation from self-consistent semiclassical gravity, Phys. Rev. D 45, 1953 (1992).

[5] K. S. Stelle, Classical gravity with higher derivatives, Gen. Relativ. Gravit. 9, 353 (1978).

[6] G. T. Horowitz and R. M. Wald, Dynamics of Einstein's equation modified by a higher-order derivative term, Phys. Rev. D 17, 414 (1978).

[7] P. Dirac, Classical theory of radiating electrons, Proc. R. Soc. London, Ser. A 167, 148 (1938).

[8] H. J. Bhabha, On the expansibility of solutions in powers of the interaction constants, Phys. Rev. 70, 759 (1946).

[9] L. D. Landau and E. M. Lifshitz, The classical theory of fields, fourth edition (Butterworth-Heinemann, Oxford, England, 2000).

[10] L. Neslusan, "On the global electrostatic charge of stars", Astronomy and Astrophysics, v.372, p.913-915 (2001)

[11] A. Ori and E. Rosenthal, Universal self-force from an extended object approach, Phys. Rev. D 68, 041701 (2003); A. Ori and E. Rosenthal, Calculation of the self force using the extended-object approach, J. Math. Phys. 45, 2347 (2004).

[12] S. M. Christensen, Vacuum expectation value of the stress tensor in an arbitrary curved background: The covariant pointseparation method, Phys. Rev. D 14, 2490 (1976).

[13] S. M. Christensen, Regularization, renormalization, and covariant geodesic point separation, Phys. Rev. D 17, 946 (1978).

[14] R. M. Wald, Quantum field theory in curved spacetime and black hole thermodynamics (The University of Chicago Press, Chicago, 1994), p. 99.

[15] C. Teitelboim, D. Villarroel, and C. G. Van Weert, Classical electrodynamics of retarded fields and point particles, Riv. Nuovo Cimento 3, 9 (1980).

[16] G. W. Ford and R. F. O'Connell, Radiation reaction in electrodynamics and the elimination of runaway solutions, Phys. Lett. A 157, 217 (1991).

[17] L. Bel and H. S. Zia, Regular reduction of relativistic theories of gravitation with a quadratic Lagrangian, Phys. Rev. D 32, 3128 (1985).

[18] J. Z. Simon and L. Parker, Einstein equation with quantum corrections reduced to second order, Phys. Rev. D 47, 1339 (1993).

[19] A. Lanir and O. Sela, Curing the Self-Force Runaway Problem in Finite-Difference Integration, Phys. Rev. D 99, 064031 (2019).

[20] G. T. Horowitz, Semiclassical relativity: The weak-field limit, Phys. Rev. D 21, 1445 (1980). 